\title{
Design Equations for Closely Spaced Two-Element Interferometer for Radio Cosmology
}

\author{
Adrian T. Sutinjo, Benjamin McKinley, Leonid Belostotski, Daniel C. X. Ung, and Jishnu \\ Nambissan $T$.
}

\begin{abstract}
Closely spaced interferometers are useful for observing highly diffuse radio sources such as the Milky Way and the global cosmological signal. We develop a simple equivalent circuit to capture key interactions between the design parameters that influence systematic errors such as mutual coupling, low noise amplifier (LNA) noise coupling, ground noise effect, and cross talk. These are key engineering considerations that have not received in-depth treatment in the context of interferometric cosmological signal detection. We discuss a realistic example of closely spaced horizontal dipoles over a lossy ground for cosmological signal detection from $50 \mathrm{MHz}$ to 100 MHz. This reveals the level of attention required to mitigate these sources of systematic errors.
\end{abstract}

\section{Introduction}

The angular resolution of an interferometer is inversely proportional to the separation between the elements. The more extended the sources, the closer the spacings required for observation. In radio cosmology, the signal of interest is a very small perturbation on the order of 10 's $\mathrm{mK}$ to 100 's $\mathrm{mK}$ in the Cosmic Microwave Background (CMB) of $3 \mathrm{~K}$ in the frequency range of approximately $20 \mathrm{MHz}$ to $200 \mathrm{MHz}$ [1]. This signal is expected to be present isotropically in the entire sky, hence the term global signature [1, 2]. Therefore the spacing required for observation is extremely small, much less than what was considered short spacing for dish-based radio interferometric imaging [3, 4]. Indeed, early experiments and the recent claimed detection of such signal used a single antenna radiometer (zero spacing) in the $50 \mathrm{MHz}$ to $100 \mathrm{MHz}$ range [5-7].

The interest now is to verify the claimed detection using a different approach to avoid similar systematic effects, hence the idea of using a close-spacing interferometer [8-12]. However, extrapolation of radio interferometry equations [13], which are based on geometry, to very close spacings must be approached with caution. At subwavelength spacings, the electromagnetic coupling between the antennas is no longer

Manuscript received 15 July 2020.

Adrian T. Sutinjo, Benjamin McKinley, Daniel C. X. Ung, and Jishnu Nambissan T. are with ICRAR/Curtin University, 1 Turner Avenue, Bentley, WA 6102, Australia; e-mail: adrian.sutinjo@ curtin.edu.au.

Leonid Belostotski is with the Department of Electrical and Computer Engineering, University of Calgary, 2500 University Drive NW, Calgary, AB T2N 1N4, e-mail: lbelosto@ucalgary.ca. negligible. Most importantly, this coupling permits a leakage path of what is otherwise uncorrelated LNA noise to the neighboring antenna, leading to a spurious noise correlation. This process in referred to as internal noise coupling. This is particularly critical since the primary argument in favor of the closely spaced interferometer in [8-11] is that the internal noise either does not correlate or does so negligibly. In this article, we demonstrate a design method for a very closely spaced two-element interferometer that includes noise coupling, ohmic loss, and cross talk.

\section{Background and Theory}

Figure 1 depicts the two-element interferometer. It consists of two closely spaced antennas, each connected to an LNA. The antennas form a coupled system that is illuminated by the external signals, which include farfield sky signal and near-field noise due to ohmic losses from the environment. The far-field signals consist of foregrounds such as extra-galactic point sources and a dominant galactic noise that is $\sim 10^{2} \mathrm{~K}$ to $\sim 10^{4} \mathrm{~K}$ in this frequency range. The foreground signals, however, are expected to be spectrally smooth, which allows the cosmological signal to be separated [14].

The correlation of the signals at the LNA outputs is expected to produce the desired external signal. The LNA noise is uncorrelated so the expected contribution is negligible, unlike the single antenna radiometer in which the LNA noise is dominant. This expectation is an important aspect of our review.

Figure 2 is a complete schematic representation of the two-element interferometer in which the noise sources are separated from the noiseless components $[15,16]$. The antenna network consists of a noiseless $T$ network representing reciprocal antennas. The desired global signal is contained in the correlation of the external noise sources, $\left\langle V_{e 1} V_{e 2}^{*}\right\rangle$, seen at the antenna ports. The internal voltage and current noise sources $\left(V_{n 1}, V_{n 2}, I_{n 1}, I_{n 2}\right)$ are due to the LNAs and noise in both correlator channels. The noise sources with the same subscript ${ }_{n 1}$ or ${ }_{n}$ 2 are partially correlated; the noise sources with different subscripts do not correlate. Measurement of $\left\langle V_{e 1} V_{e 2}^{*}\right\rangle$ must be inferred from the correlation of voltages across the LNA inputs, $\left\langle V_{L 1} V_{L 2}^{*}\right\rangle$.

\subsection{External Noise Sources}

The mutual coherence of $V_{e 1}$ and $V_{e 2}$ due to unpolarized far-field noise sources characterized by noise temperature $T_{\text {ext }}(\theta, \phi)$ in the spherical coordinate 


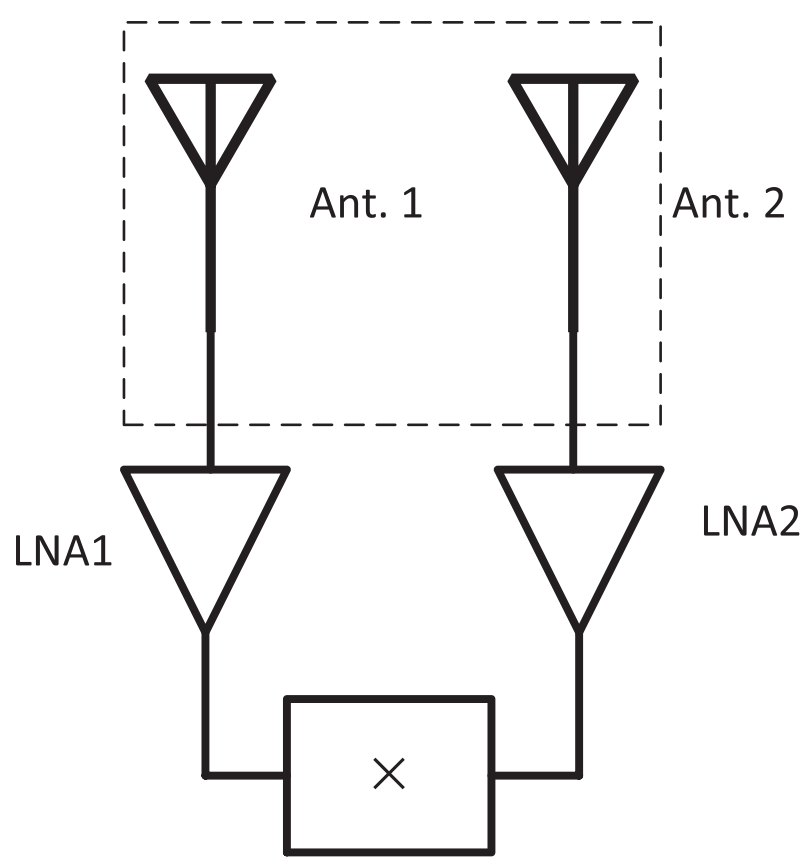

Figure 1. The block diagram of a two-element interferometer. The LNA outputs enter into the correlator, and observation is made using the correlator output.

system is [17-20]

$$
\frac{\left\langle V_{e 1} V_{e 2}^{*}\right\rangle_{f f}}{\Delta f}=\frac{\eta_{0} k}{\lambda^{2}} \int_{0}^{2 \pi} \int_{0}^{\pi} T_{\text {ext }}(\theta, \phi) \mathbf{l}_{1} \cdot \mathbf{l}_{2}^{*} \sin \theta d \theta d \phi
$$

where $\mathbf{l}_{1,2}=\hat{\theta} l_{1,2}^{\theta}(\theta, \phi)+\hat{\phi} l_{1,2}^{\phi}(\theta, \phi)$ is the open-circuit effective antenna length, $\eta_{0}=\sqrt{\mu_{0} / \epsilon_{0}} \approx 120 \pi \Omega$ is the free space impedance, $k$ is the Boltzmann constant, and $\lambda$ is the wavelength at the center frequency of the observation and $\Delta f$ is the frequency bandwidth of the observation. The open-circuit antenna effective lengths $\mathbf{l}_{1}, \mathbf{l}_{2}$ are taken with respect to a common coordinate origin $(0,0)$ in the presence of the other element, which is open-circuited at its port.

The global signal can be modeled as an isotropic far-field noise $T_{\text {ext }}(\theta, \phi)=T_{\text {iso }}$ such that it moves out of the integral sign in (1). As first-order approximation, it is helpful to initially assume that all external noise sources are at thermal equilibrium and/or ohmic losses are negligible. In this case,

$$
\begin{aligned}
& \left\langle V_{e 1} V_{e 2}^{*}\right\rangle_{\text {iso }}=4 k T_{\text {iso }} \Delta f R_{12} \\
& \left\langle\left|V_{e 1}\right|^{2}\right\rangle_{\text {iso }}=4 k T_{\text {iso }} \Delta f R_{11}
\end{aligned}
$$

where $R_{11}$ is the antenna 1 self-resistance and $R_{12}$ is the antenna mutual resistance.

Assuming foreground subtraction is successful, $T_{\text {iso }}$ is the CMB temperature. The detection process, then, involves removal of the $R_{12}$ scaling factor from (2) and searching for the 10 's $\mathrm{mK}$ to 100 's $\mathrm{mK}$ perturbation

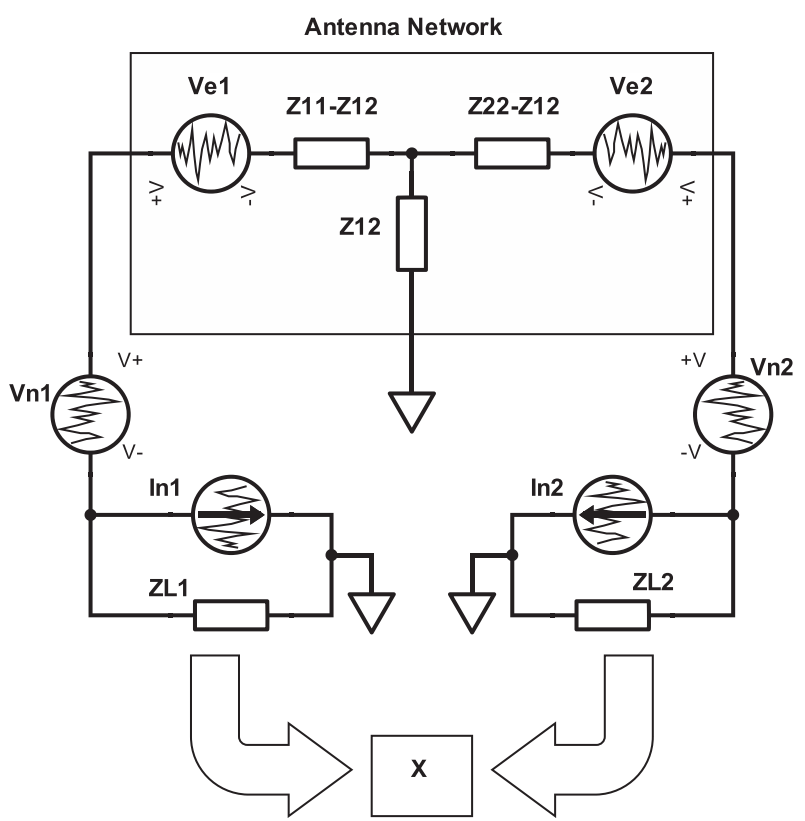

Figure 2. Equivalent circuit for a two-element interferometer including external $\left(V_{e 1}, V_{e 2}\right)$ and internal noise sources $\left(V_{n 1}, V_{n 2}\right.$, $\left.I_{n 1}, I_{n 2}\right)$. The antennas are reciprocal $Z_{21}=Z_{12} ; Z_{L 1}, Z_{L 2}$ are the LNA input impedances; the voltages across the LNA inputs are $V_{L 1}, V_{L 2}$.

in $T_{\text {iso }}$ in the frequency band of interest. Therefore, it is important that frequency variation of $R_{12}$ be smooth; sharp turns and zero crossings are generally undesirable. The quantity $R_{12}$ is very well known and has been studied in the antenna community for many decades. The results have been tabulated and plotted [21-23], and $R_{12}$ is now easily computed using electromagnetic simulation as well as measured using a vector network analyzer (VNA).

\subsection{Internal Noise Sources}

The noise of an active two-port is usually given in data sheets as four noise parameters: $R_{n}$ (noise resistance, $\Omega$ ), $F_{\min }$ (minimum noise factor, linear), and $Y_{\text {opt }}$ (optimum source admittance, S), which are convertible as follows [16]:

$$
\begin{gathered}
\left\langle\left|V_{n}\right|^{2}\right\rangle=4 k T_{0} \Delta f R_{n} \\
\left\langle V_{n} I_{n}^{*}\right\rangle=4 k T_{0} \Delta f\left(\frac{F_{\text {min }}-1}{2}-R_{n} Y_{\mathrm{opt}}^{*}\right) \\
\left\langle\left|I_{n}\right|^{2}\right\rangle=4 k T_{0} \Delta f R_{n}\left|Y_{\mathrm{opt}}\right|^{2}
\end{gathered}
$$

where $T_{0}=290 \mathrm{~K}$ is the reference temperature. As an example, a pHEMT ultra LNA SAV-541+ (https:// www.minicircuits.com/pdfs/SAV-541+.pdf) from Minicircuits at $V_{\mathrm{DS}}=3 \mathrm{~V}, I_{D}=60 \mathrm{~mA}$ bias at $500 \mathrm{MHz}$ (lowest available frequency data) has $R_{n}=2.5 \Omega, Y_{\text {opt }}=$ $0.0106-\mathrm{j} 0.0017 \mathrm{~S}$, and $F_{\min }=1.025$. 


\section{Correlated Products}

\subsection{Response to External Noise}

For design equations, it is adequate to consider identical antennas (such that $Z_{L 1}=Z_{L 2}=Z_{L}$; $Z_{11}=Z_{22}$ ) and the LNA noise statistics are identical. The embedded antenna impedance is the impedance looking into the antenna port $(\|$ indicates parallel connection) $Z_{\text {emb }}=Z_{11}-Z_{12}+Z_{12} \|\left(Z_{11}-Z_{12}+Z_{L}\right)$. With these assumptions and using circuit analysis based on Figure 2, we obtain the correlation product seen at the LNA inputs:

$$
\begin{aligned}
\frac{\left\langle V_{L 1} V_{L 2}^{*}\right\rangle_{\mathrm{ext}}}{|\chi|^{2}}= & -2 \Re(\varphi)\left\langle\left|V_{e 1}\right|^{2}\right\rangle+\left\langle V_{e 1} V_{e 2}^{*}\right\rangle \\
& +|\varphi|^{2}\left\langle V_{e 1}^{*} V_{e 2}\right\rangle
\end{aligned}
$$

where the voltage division ratios are $\varphi=Z_{12} /$ $\left(Z_{11}+Z_{L}\right), \quad \chi=Z_{L} /\left(Z_{L}+Z_{\mathrm{emb}}\right) . \varphi$ converts a series voltage source from one side of the $\mathrm{T}$ network to a Thévenin equivalent source as seen by the load on the opposite side; $\chi$ is the voltage division ratio between the voltage at the load and the Thévenin equivalent source. As $Z_{L} \rightarrow \infty, \varphi \rightarrow 0, \chi \rightarrow 1$ such that $\left\langle V_{L 1} V_{L 2}^{*}\right\rangle_{\text {ext }} \rightarrow$ $\left\langle V_{e 1} V_{e 2}^{*}\right\rangle$, which is the ideal result. Finite $Z_{L}$ and $\varphi$ lead to extra terms on the right-hand side of (7) that must be known and removed from the measurement. The presence of $Z_{12}$ in $\varphi$, in which $R_{12}$ is required for isotropic noise correlation in (2), inevitably produces measurement artifacts.

\subsection{Response to Internal Noise}

The mutual coherence of LNA input voltages due to internal noise is

$$
\frac{\left\langle V_{L 1} V_{L 2}^{*}\right\rangle_{\text {int }}}{|\chi|^{2}}=2 \Re\left(\varphi \vartheta_{n}\right)
$$

where

$$
\begin{aligned}
\vartheta_{n}= & -\left\langle\left|V_{n}\right|^{2}\right\rangle+\left\langle\left|I_{n}\right|^{2}\right\rangle Z_{\mathrm{emb}}^{*} Z_{L}+\left\langle V_{n}^{*} I_{n}\right\rangle Z_{L} \\
& -\left\langle V_{n} I_{n}^{*}\right\rangle Z_{\mathrm{emb}}^{*}
\end{aligned}
$$

Again, the presence of $Z_{12}$ in $\varphi$ produces artifacts. Note that $Z_{L} \rightarrow \infty, \varphi \rightarrow 0$ does not imply vanishing mutual coherence due to internal noise because of the presence of the noise current $\left\langle\left|I_{n}\right|^{2}\right\rangle$ in (9). Zero mutual coherence due to internal noise is possible, but the condition is restrictive and unlikely to be met over the required bandwidth. For example, one possibility is $\vartheta_{n}$ $=0$. A solution for this can be obtained by substituting (4) to (6) into (9) and setting it to zero, which results in $Z_{L}^{*}=Z_{\mathrm{emb}}=Z_{\mathrm{opt}}=Y_{\mathrm{opt}}^{-1}$, which is a tight constraint.

For a two-element system, we find the form of (8) and (9) more intuitive and convenient for design as it is based on schematic and involves no matrix inversion. These equations have been numerically validated against a different formulation in [18]. We found

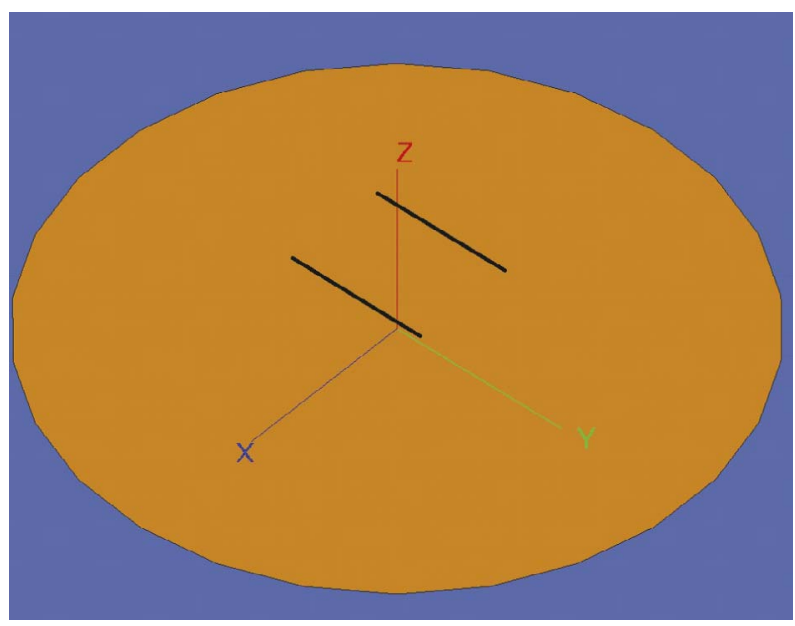

Figure 3. Two closely spaced parallel dipoles over ground. The dipole length is $L=1.3 \mathrm{~m}$, wire radii $1 \mathrm{~mm}$, separation $d=1 \mathrm{~m}$, and height above ground $h=0.7 \mathrm{~m}$. The ground plane is a circular perfect electric conductor (PEC), which for clarity is shown as $6 \mathrm{~m}$ in diameter. The ground plane is placed over a soil model based on a Murchison Radio-astronomy Observatory (MRO) sample with 2\% humidity [24]. The dipoles are PEC.

identical results for two parallel thin dipoles $(1.44 \mathrm{~m}$ long, $0.9 \mathrm{~m}$ apart in free space) from $50 \mathrm{MHz}$ to 100 $\mathrm{MHz}$ (not shown) connected to SAV-541 LNAs. Both methods predict mutual coherence zero crossing due to internal noise at approximately $99 \mathrm{MHz}$. This confirms both the existence of vanishing internal noise mutual coherence as well as its narrow band nature.

\section{Two Parallel Dipoles Over a Ground Plane}

Figure 3 depicts an example of a two-dipole closely spaced interferometer for global signal detection in $50 \mathrm{MHz}$ to $100 \mathrm{MHz}$. Any earth-bound system is a half-space system and must contend with soil losses. We selected horizontal dipoles as opposed to monopoles because the former is far more amenable to effective shielding from the soil. For example, the horizontal dipole system can achieve radiation efficiency $\geq 95 \%$ while the corresponding monopole system efficiency is approximately $50 \%$. However, the tradeoff is the dipole image due the PEC ground plane reduces $R_{11}$ and $R_{12}$, especially at $50 \mathrm{MHz}$. In this system, the onset of $R_{12}$ zero crossing at high frequency places an upper limit on the dipole spacing. We select the spacing to prevent abrupt change in $R_{12}$ throughout the desired band.

\subsection{Noise From Ohmic Loss}

In the presence of ohmic loss, the external noise sources comprise two components due to the far-field sources and ohmic loss, e.g., $V_{e 1}=V_{e 1: f f}+V_{e 1: \Omega}$ and similarly for $V_{e 1}$. The noise from ohmic loss is uncorrelated with the far-field sources. The mutual coherence of the external noise voltages due to the ohmic losses, $V_{e 1: \Omega}$ and $V_{e 2: \Omega}$, is obtained by placing the 


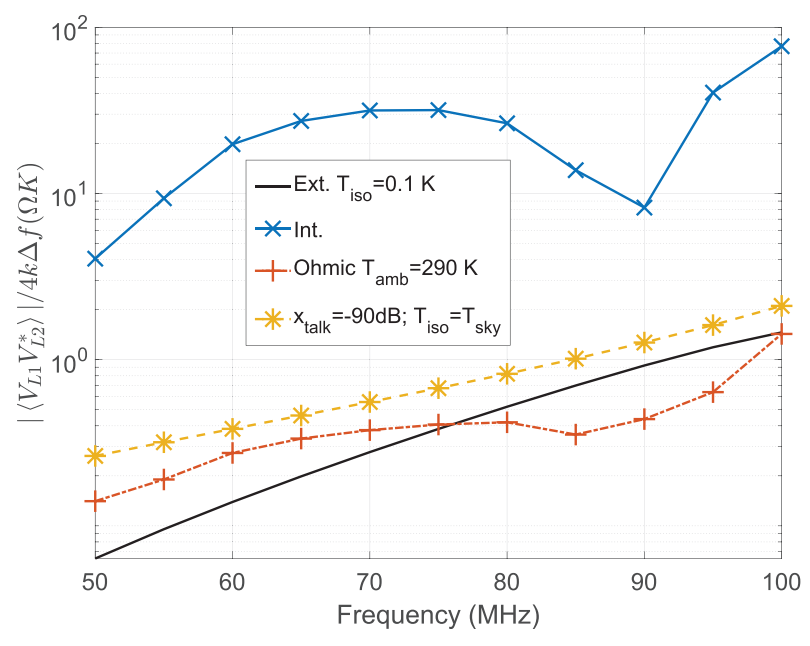

Figure 4. Calculated mutual coherence (absolute value) due to internal noise, isotropic sky at $0.1 \mathrm{~K}$, ohmic loss, and cross talk assuming $x_{\text {talk }}=-90 \mathrm{~dB}$ for the two-dipole system in Figure 3 with ground plane diameter of $40 \mathrm{~m}$.

antenna system in a test thermal equilibrium environment of ambient temperature $T_{\mathrm{amb}}$ and taking the difference

$$
\begin{aligned}
\frac{\left\langle V_{e 1: \Omega} V_{e 2: \Omega}^{*}\right\rangle}{4 k T_{\mathrm{amb}} \Delta f} & =R_{12}-\frac{\eta_{0}}{4 \lambda^{2}} \int_{0}^{2 \pi} \int_{0}^{\pi / 2} \mathbf{l}_{1} \cdot \mathbf{l}_{2}^{*} \sin \theta d \theta d \phi \\
& \triangleq \Delta R_{12}
\end{aligned}
$$

and similarly for $\left\langle\left|V_{e 1: \Omega}\right|^{2}\right\rangle$ by replacing the subscript $\cdot 2$ with ..$_{1}$ in (10); the result is $\Delta R_{11}$. The resulting quantities below are substituted into the external noise correlation equation (7):

$$
\begin{aligned}
& \left\langle\left|V_{e 1}\right|^{2}\right\rangle=\left\langle\left|V_{e 1: \mathrm{ff}}\right|^{2}\right\rangle+4 k T_{\mathrm{amb}} \Delta R_{11} \Delta f \\
& \left\langle V_{e 1} V_{e 2}^{*}\right\rangle=\left\langle V_{e 1: f f} V_{e 2: f f}^{*}\right\rangle+4 k T_{\mathrm{amb}} \Delta R_{12} \Delta f
\end{aligned}
$$

\subsection{Cross-talk Consideration}

In Figure 2, cross talk represents the amount of $V_{L 1}$ that leaks to the input of the other LNA and vice versa. The leakage scaling factor that multiplies $V_{L 1}$ is the cross-talk factor $x_{\text {talk. }}$. It can be shown that $x_{\text {talk }}$ produces an additive term in the right-hand side of (7):

$$
\Delta_{x}=-2 \Re\left(x_{\text {talk }}\right)\left\langle\left|V_{e 1}\right|^{2}\right\rangle+\left\langle V_{e 1}^{*} V_{e 2}\right\rangle\left|x_{\text {talk }}\right|^{2}
$$

The first term in the right-hand side of (7) represents the leakage of the single-element response to the desired product. This is the primary impact of cross talk that must be carefully managed because the foreground sky under observation is a few orders of magnitude higher than $0.1 \mathrm{~K}$.

\subsection{Simulated Performance}

Figure 4 shows the overall performance of the two-dipole system simulated using FEKO. The noise due to ohmic losses is calculated for $T_{\mathrm{amb}}=T_{0}$. The antenna system is placed under isotropic sky with $T_{\text {sky }}=$ $60 \lambda^{2.55} \mathrm{~K}$, which models the exponential sky noise increase with increasing wavelength [24]. The ohmic loss contribution is computed numerically with far-field integrals samples at $2^{\circ}$ resolution. We studied the effect of soil noise as a function of ground plane diameter from $6 \mathrm{~m}$ to $40 \mathrm{~m}$. As expected, the ohmic noise contribution decreased with increasing ground plane diameter. At $40 \mathrm{~m}$ diameter, the level of mutual coherence due to ohmic loss becomes comparable to the response of the system observing $0.1 \mathrm{~K}$ isotropic sky. The effect of constant cross-talk level of $-90 \mathrm{~dB}$ over frequency produces mutual coherence at a level comparable to that of an isotropic sky at a few hundred $\mathrm{mK}$. This suggests significant isolation and shielding is required between the two branches of circuitry connected to the antennas.

The LNAs are the same part as in Sec. 2.2 with data sheet values extrapolated to $50 \mathrm{MHz}$ by inferring the trend of the lowest few values. The input of the LNA may be modeled as an $11 \Omega$ resistor in series with a $5 \mathrm{pF}$ capacitor. The noise parameters are the same as the $500 \mathrm{MHz}$ value except for $B_{\text {opt }}=(-0.0017 \mathrm{~S}) f_{\mathrm{MHz}} /$ 500. Figure 4 shows that contribution of internal noise, at up to two orders of magnitude above the desired response, is the highest contributor to systematic error. This suggests that calibration of internal noise coupling is essential for close-spacing interferometry for cosmological signal detection.

\section{Conclusion}

We examined the closely spaced interferometer system through design formulas and an equivalent circuit. The results show that it is possible to design a closely spaced two-element interferometer with spectrally smooth response that is sensitive to the highly diffuse source over $50 \mathrm{MHz}$ to $100 \mathrm{MHz}$ for cosmological signal detection. Mutual coherence due to internal noise coupling is an inherent property of a closely spaced interferometer as the mutual resistance that produces the zero-spacing response also couples the internal noise. Contributions due to ohmic loss and cross talk are also important, and mitigation entails large ground plane ( $\sim 10$ 's $\mathrm{m}$ diameter) and aggressive shielding.

\section{References}

1. J. R. Pritchard and A. Loeb, "21 cm Cosmology in the 21st Century," Reports on Progress in Physics, 75, July 2012, p. 086901.

2. P. A. Shaver, R. A. Windhorst, P. Madau, and A. G. de Bruyn, "Can the Reionization Epoch Be Detected as a Global Signature in the Cosmic Background?," Astronomy and Astrophysics, 345, May 1999, pp. 380-390.

3. R. Ekers and A. Rots, "Short Spacing Synthesis from a 
Primary Beam Scanned Interferometer," International Astronomical Union Colloquium, 49, 1979, pp. 61-63.

4. R. Braun and R. A. M. Walterbos, "A Solution to the Short Spacing Problem in Radio Interferometry," Astronomy \& Astrophysics, 143, February 1985, pp. 307-312.

5. A. E. E. Rogers and J. D. Bowman, "Absolute Calibration of a Wideband Antenna and Spectrometer for Accurate Sky Noise Temperature Measurements," Radio Science, 47, 6, 2012, pp. 1-9.

6. J. D. Bowman, A. E. E. Rogers, R. A. Monsalve, T. J. Mozdzen, and N. Mahesh, "An Absorption Profile Centred at 78 Megahertz in the Sky-Averaged Spectrum," Nature, 555, March 2018, pp. 67-70.

7. R. A. Monsalve, A. E. E. Rogers, J. D. Bowman, and T. J. Mozdzen, "Calibration of the EDGES High-Band Receiver to Observe the Global $21 \mathrm{~cm}$ Signature from the Epoch of Reionization," Astrophysical Journal, 835, January 2017, https://doi.org/10.3847/1538-4357/835/1/ 49.

8. M. E. Presley, A. Liu, and A. R. Parsons, "Measuring the Cosmological $21 \mathrm{~cm}$ Monopole with an Interferometer," Astrophysical Journal, 809, August 2015, https://doi.org/ 10.1088/0004-637X/809/1/18.

9. S. Singh, R. Subrahmanyan, N. U. Shankar, and A. Raghunathan, "On the Detection of Global 21-cm Signal From Reionizaton Using Interferometers," Astrophysical Journal, 815, December 2015, https://doi.org/10.1088/ 0004-637X/815/2/88

10. N. Mahesh, R. Subrahmanyan, N. Udaya Shankar, and A. Raghunathan, "A Wideband Resistive Beam-Splitter Screen," IEEE Transactions on Antennas and Propagation, 63, November 2015, pp. 4835-4847.

11. T. Venumadhav, T.-C. Chang, O. Doré, and C. M. Hirata, "A Practical Theorem on Using Interferometry to Measure the Global $21 \mathrm{~cm}$ Signal," Astrophysical Journal, 826, July 2016, https://doi.org/10.3847/0004637X/826/2/116.

12. B. McKinley, C. M. Trott, M. Sokolowski, R. B. Wayth, A. Sutinjo, et al., "The All-Sky SignAl Short-Spacing INterferometer (ASSASSIN) I: Global Sky Measurements With the Engineering Development Array-21," Monthly Notices of the Royal Astronomical Society, 499, 1, November 2020, pp. 52-67, https://doi.org/10.1093/ mnras/staa2804.

13. A. R. Thompson, J. M. Moran, and G. W. Swenson Jr.,
Interferometry and Synthesis in Radio Astronomy, 3rd Edition, New York, Springer, 2017.

14. N. Petrovic and S. P. Oh, "Systematic Effects of Foreground Removal in 21-cm Surveys of Reionization," Monthly Notices of the Royal Astronomical Society, 413, May 2011, pp. 2103-2120.

15. H. Rothe and W. Dahlke, "Theory of Noisy Fourpoles," Proceedings of the IRE, 44, June 1956, pp. 811-818.

16. H. Hillbrand and P. Russer, "An Efficient Method for Computer Aided Noise Analysis of Linear Amplifier Networks," IEEE Transactions on Circuits and Systems, 23, April 1976, pp. 235-238.

17. D. C. X. Ung, M. Sokolowski, A. T. Sutinjo, and D. B. Davidson, "Noise Temperature of Phased Array Radio Telescope: The Murchison Widefield Array and the Engineering Development Array," IEEE Transactions on Antennas and Propagation, 68, 7, 2020, pp. 53955404.

18. K. F. Warnick, R. Maaskant, M. V. Ivashina, D. B. Davidson, and B. D. Jeffs, Phased Arrays for Radio Astronomy, Remote Sensing, and Satellite Communications, Cambridge, United Kingdom, Cambridge University Press, 2018.

19. K. F. Warnick and B. D. Jeffs, "Efficiencies and System Temperature for a Beamforming Array," IEEE Antennas and Wireless Propagation Letters, 7, 2008. pp. 565-568.

20. K. F. Warnick, B. Woestenburg, L. Belostotski, and P. Russer, "Minimizing the Noise Penalty Due to Mutual Coupling for a Receiving Array," IEEE Transactions on Antennas and Propagation, 57, 6, June 2009, pp. 16341644.

21. G. H. Brown and R. King, "High-Frequency Models in Antenna Investigations," Proceedings of the Institute of Radio Engineers, 22, April 1934, pp. 457-480.

22. N. Alexopoulos and I. Rana, "Mutual Impedance Computation Between Printed Dipoles," IEEE Transactions on Antennas and Propagation, 29, January 1981, pp. 106-111.

23. W. L. Stutzman and G. A. Thiele, Antenna Theory and Design, 3rd Edition, Hoboken, NJ, Wiley, 2013, Chapter 8.

24. A. T. Sutinjo, T. M. Colegate, R. B. Wayth, P. J. Hall, E. de Lera Acedo, et al., "Characterization of a LowFrequency Radio Astronomy Prototype Array in Western Australia," IEEE Transactions on Antennas and Propagation, 63, December 2015, pp. 5433-5442. 